\title{
A Cotton Irrigator's Decision Support System and Benchmarking Tool Using National, Regional and Local Data
}

\author{
Jamie Vleeshouwer ${ }^{1}$, Nicholas J. Car ${ }^{1}$, and John Hornbuckle ${ }^{2}$ \\ ${ }^{1}$ Land and Water Flagship: CSIRO, Brisbane, QLD Australia \\ Jamie.Vleeshouwer@csiro.au \\ ${ }^{2}$ Agriculture Flagship: CSIRO, Griffith, NSW Australia
}

\begin{abstract}
We are developing a smart phone application that provides irrigation water management advice using satellite imagery, weather stations and fieldscale farmer provided data.

To provide tailored advice we use high resolution satellite imagery with national coverage provided by Google Earth Engine services to estimate fieldspecific crop growth information - crop coefficients - and we are among the first systems to do so. These coefficients combined with regional scale weather station data for major cotton growing regions and farmer-supplied data means we can run daily water balance calculations for every individual cotton field in Australia and provide irrigation decision support advice.

We are using automated data processing to ensure the latest satellite and weather data is used for advice without manual effort.

We will also deliver benchmarking data to farmers based on their previous seasons as well as peers' farms in order to compare absolute (calculated) and relative (benchmarked) advice.
\end{abstract}

Keywords: irrigation, satellite data, weather station, mobile phone app, evapotranspiration.

\section{Background}

IrriSAT is a weather based irrigation scheduling service which is used to inform farmers how much water their crop has used and how much how much irrigation they need to apply. Information is produced daily, and can work with different crop types, across large spatial scales. The system is being developed by the Commonwealth and Industrial Research Organisation (CSIRO) for the Cotton Research and Development Corporation (CRDC).

The system uses satellite images to determine the Normalized Difference Vegetation Index (NDVI) for each field, from which the plant canopy size can be determined and a specific crop coefficient (Kc) can be estimated (Hornbuckle et al, 2009). By combining Kc with daily reference Evapotranspiration (ET0) observations from a nearby weather station, the crop water usage can be determined and advice can be provided regarding the amount of irrigation to be applied. 
Successful trials of the service have previously been undertaken with grape and citrus irrigators, leading to further trials with cotton in the Gwydir, Border Rivers and Lower Namoi valley areas from 2009 - 2012. In the final season of these trials, 72 irrigators were using the service, whereby the preferred interface for interacting with the service was via SMS messages. (Car et al 2012). The SMS interface allowed an irrigator to enter codes which represented their identity and their fields, along with how much irrigation had been applied to the field (from observed rainfall, or applied by an irrigation system). This information was then used to calculate the daily water balance for their field and provide a response via SMS with the water deficit to aid with managing irrigation schedules.

Whilst the SMS interface provided an efficient way for irrigators to register their data with the IrriSAT service, it was also subject to data entry errors such as codes / water quantities often being mistyped (either by miskeying, or the mobile phone's autocorrect features modifying the inputs). Recent investments will allow the technology overcome these issues and to be further developed for the next generation of services which include: automated near-real time forecasting; regional crop water use productivity benchmarking, and in season yield production. To better deliver these services to irrigator's, smart phone and tablet applications will be developed, along with the required automated data feeds in standardized exchange formats to drive the next generation IrriSAT applications.

\section{The IrriSAT Concept}

Rule-based irrigation advice is generated using data from sources shown in Fig. 1 according to the equation (1). The water balance for a crop is determined by representing the field as a closed-system summing the daily calculated water balance of the field which is determined by equation (2).

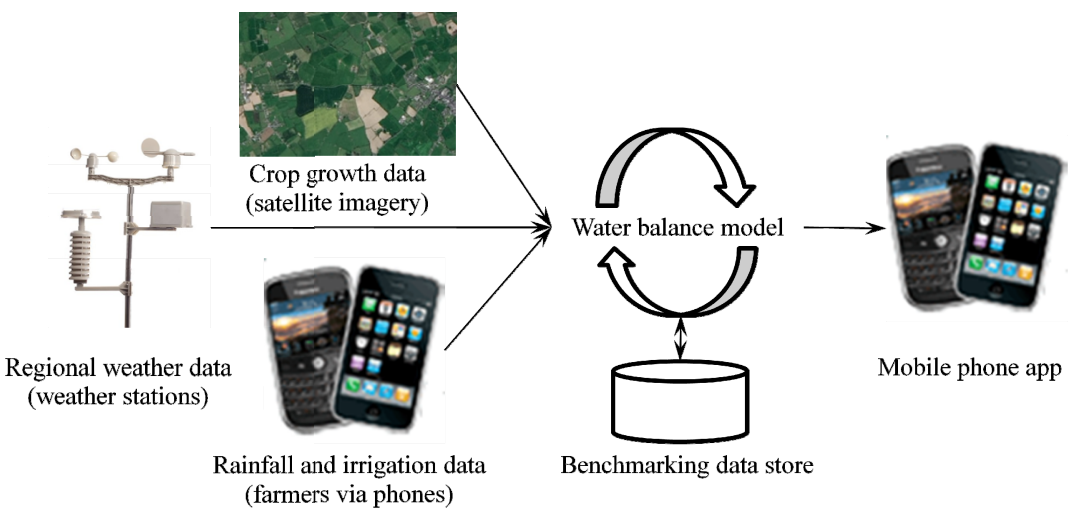

Fig. 1. A basic information flow diagram of the IrriSAT system

The water balance is calculated by subtracting crop evapotranspiration, calculated by reference evapotranspiration multiplied by a crop coefficient (for cotton), from water 
inputs (irrigation and rainfall). Whilst irrigation and rainfall simply can be obtained observations with appropriate shaping (reduction) functions to cater for runoff, the crop evapotranspiration (ETc) is determined by a variant of the Penman-Montieth equation according to (Allen et al, 1998). The modification is for 'tall crops' which models cotton better than the standard for 'short crops (grass).

$$
\begin{gathered}
W B_{d}=\sum_{i}^{d} W B_{i} \\
d(\text { day in season }) \in[1-n] \\
W B d=\text { total season waterbalance to dayd } \\
W B_{i}=S h_{I} I_{i}+S h_{R} R_{i}-\left(E T_{0} K_{c}\right) \\
W B_{i}=\text { daily waterbalance } \\
S h_{I}=\text { shaping function for rainfall } \\
S h_{R}=\text { shaping function for rainfall } \\
E T_{0}=\text { reference evapotranspiration } \\
K_{c}=\text { crop coefficient }
\end{gathered}
$$

$W B_{d}$ is translated from millimeters depth into irrigation pump run times by knowing the application rate of the field's irrigation system. This allows a user to program a system to run in order to return $W B_{d}$ to zero (or other desired level) without further calculation.

In addition to this water balance rule-based decision support advice, the next generation of IrriSAT will deliver case-based, benchmarked decision support advice to irrigators via the methods described in Section 3.4.

\section{Establishing the Next Generation IrriSAT}

In recent years smartphones and tablets have become very popular, and we now aiming to make IrriSAT services more easily accessible through Android and iOS applications. A key factor to making these services functional are the data feeds which are required as inputs to the decision support system (DSS). These data feeds need to be: made available on demand for near real time decision support; be encoded in standardized data exchange formats; and be easily accessible over the internet via web services. Alternate forms of decision support advice are able to generated by non-rule-based artificial intelligence systems such as Case-Based reasoning. The next generation IrriSAT system will test one such form.

\subsection{Remotely Sensed Imagery}

Within previous trials of the IrriSAT service, Landsat 5 imagery was used which provided a spatial resolution of $30 \mathrm{~m}$ which was sufficient for capturing the spatial variability of the crop water use across an irrigator's field. An email would be sent to the IrriSAT administrator when new Landsat imagery was made available. The tasks the administrator would then undertake would be: 
1. Downloaded the latest imagery

2. Manually screen away the clouds based upon inspection within ERDAS IMAGINE

3. Execute an ERDAS script to produce a CSV file containing field Kc values

4. Upload the CSV to the IrriSAT database for further processing

This process was often time-consuming for the administrator, and had to be repeated approximately every 8 days as new imagery became available. However recent advancements in accessing remotely sensed data warehouses and executing scientific algorithms aim to make tasks like these mentioned more efficient. Two services currently in development include: The Australian Geoscience Data Cube (AGDC); and Google Earth Engine (GEE). Both of these services utilize supercomputing to allow users to run custom algorithms upon remotely sensed imagery via API's. Here, we have undertaken a scoping study to assess whether GEE is capable of being used for processing the modeling required for the backend IrriSAT services.

The GEE platform allows users to run algorithms on satellite imagery and earth observation data for scientific analyses and visualisation (Gorelick, 2013). It contains a petabyte-scale archive of global imagery which spans the past 40 years and uses a distributed computational model using a just-in-time approach, meaning that processing is run in real-time, however won't occur until it is required as an output or as an input to another process. This is achieved by exchanging a description of the processing activities and their sequences with GEE servers through a restful web services. Algorithms can be written using the GEE Application Programming Interface (API) which is available in both Python and Javascript programming languages.

For this study we used a combination of Landsat 8 OLI and Landsat 7 ETM+ imagery since the Landsat 5 program is no longer operational. Similarly to Landsat 5 , both Landsat $8 / 7$ exhibits a $30 \mathrm{~m}$ spatial resolution, takes 16 days to observe Earth, and is made available in 8 -day cycles. Given that Landsat 8 is offset by 8 days to Landsat 7 our approach was to combine Landsat 8/7. Whilst differences exist between the OLI and ETM+ sensors, it has been shown that the two sensors are broadly compatible across the Australian landscape with differences of approximately $5 \%$ for NDVI (Flood, 2014).

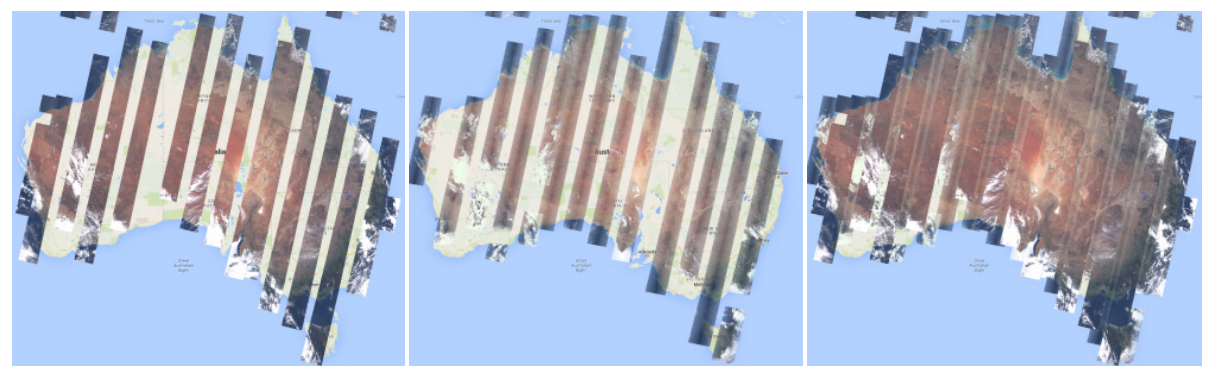

Fig. 2. Landsat 8 (left), Landsat 7 (centre) and combined Landsat $8 / 7$ (right) TOA reflectance for 16 October 2014 
The approach of combining the two datasets typically provides near full coverage for Australia every 8 days (Fig. 2), which would provide the most up to date crop water usage information to irrigators in near real time.

It is well known that the influence of cloud cover can cause remotely sensed NDVI to be underestimated (Liaw, et al 1995). In order to address this problem we are using the SimpleCloudScore algorithm which is provided within the GEE Algorithms API (Fig. 3). This algorithm works by determining a cloud-likelihood score in the range $[0,100]$ using a combination of brightness, temperature, and NDSI from the TOA reflectance imagery.

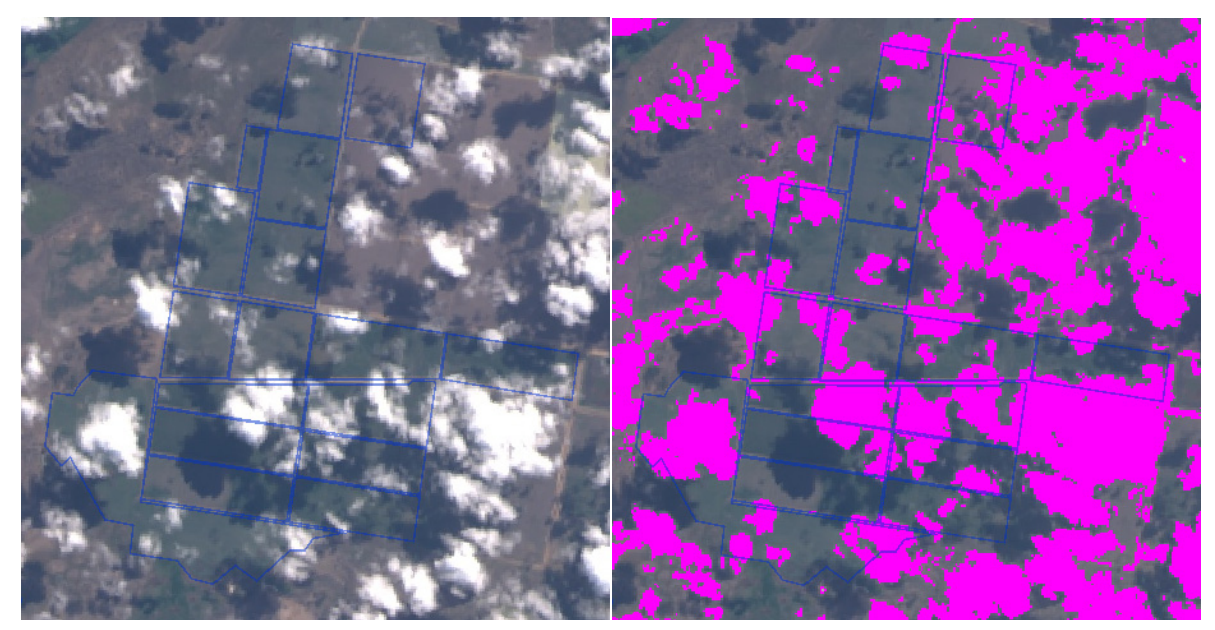

Fig. 3. Illustration of Landsat 8 TOA reflectance for 16 irrigation fields with visible cloud cover (left). The GEE SimpleCloudScore algorithm used to generate a mask for removing cloud cover from Landsat imagery (right). Detected clouds are shown in magenta.

The SimpleCloudScore algorithm is not a robust cloud detector, and is intended mainly to compare multiple looks at the same point for relative cloud likelihood, however we have found it to give adequate performance at detecting clouds when a cloud-likelihood score threshold of 17 is set. At present, an implementation of the Fmask v3.2 automated cloud detection algorithm (Z. Zhu and C.E. Woodcock, 2012) is being incorporated into the GEE API. The Fmask algorithm is an objectbased cloud detection algorithm which is able to detect clouds, cloud shadows and snow with an average overall accuracy of $96.41 \%$. When the algorithm is made available it would be useful to trial it out as cloud shadows can also lead to NDVI misinterpretations.

By combining a cloud mask with the Landsat NDVI datasets available within GEE we are then able to determine the pixels which represent a valid values. 


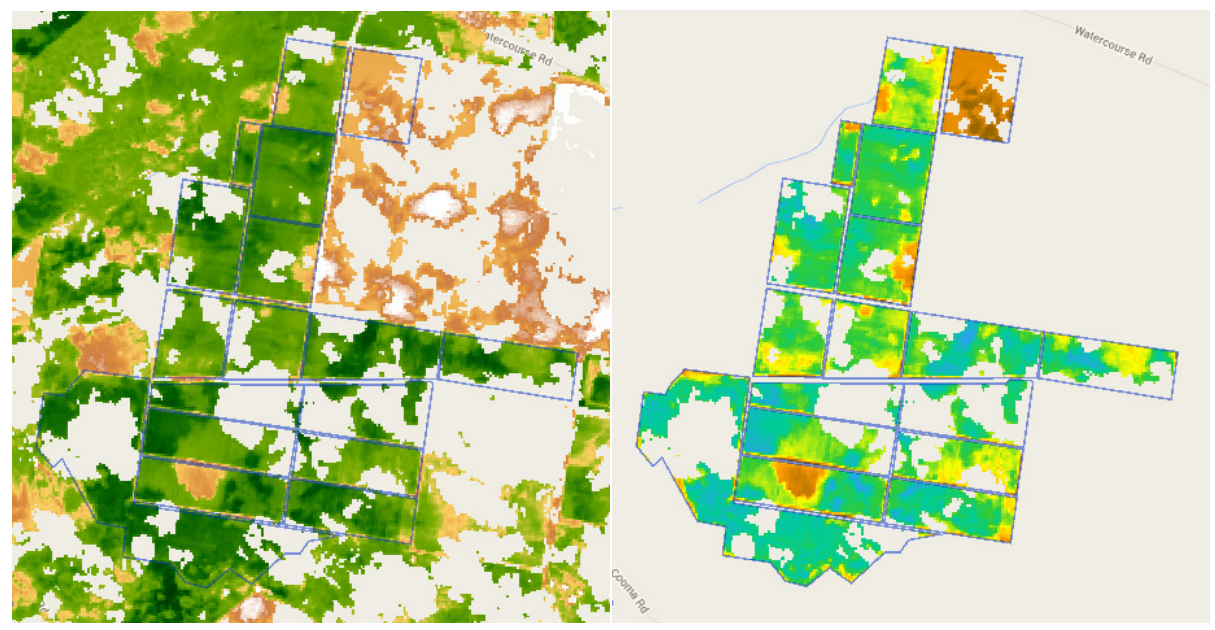

Fig. 4. Landsat 8 NDVI for the same day as is Fig. 3. The cloud mask is used the remove pixels which are determined to be invalid data (left). This image is then used to determine the crop coefficient $(\mathrm{Kc})$ for cotton using a linear scaling (right).

Examining one of these fields over the 2013-2014 cotton growing season (Fig. 5) illustrates how the crop coefficient initially starts with values of $\sim 0.1$ (bare soil) and increases to $\sim 1.0$ (grown cotton), with harvesting taking place usually at the end of the first quarter of each year.

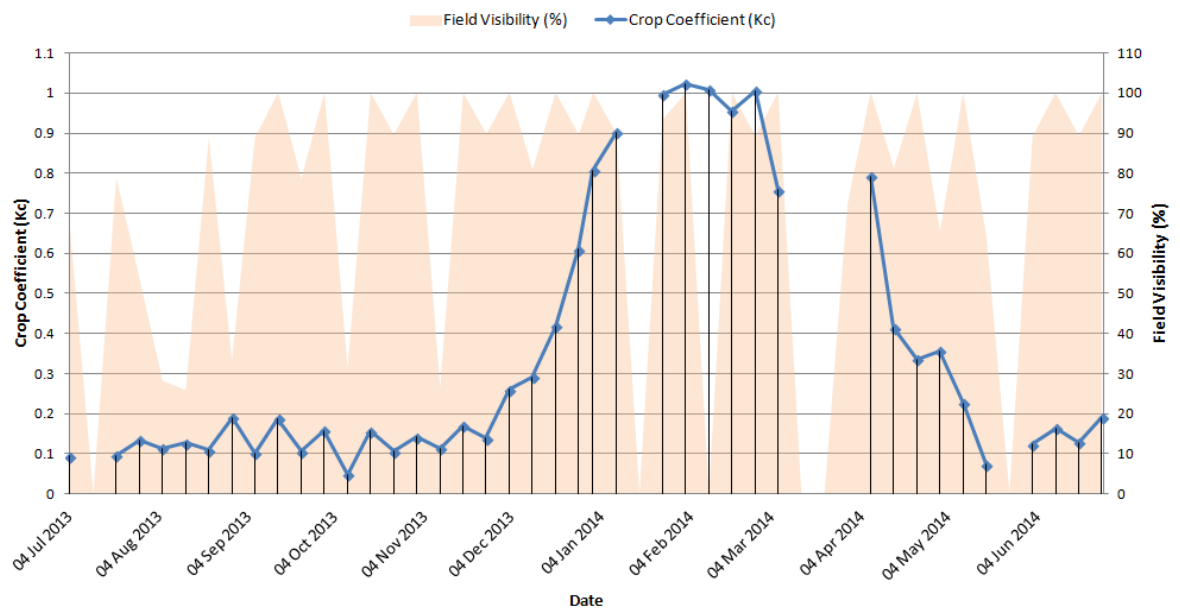

Fig. 5. Crop coefficient for an irrigators field over the 2013-2014 cotton growing season

\subsection{On-ground Weather Observations}

In order to provide regionalized evapotranspiration observations to irrigators, CSIRO maintains a network of Automatic Weather Stations (AWS). These services are currently 
tightly-coupled to the current IrriSAT system, however we will be working to expose this data via web services. This allows users or devices to be able to query and retrieve observations over the web which will play an important role for consuming data within a mobile app. To improve the accessibility of this data we are currently implementing the Sensor Observation Service (SOS) which provides a way to query real-time sensor data in a standardized data exchange. Utilising this standardized exchange format will allow us to easily expand upon our current coverage of weather stations, allowing us to query and consume SOS observations from other organisations such as the Bureau of Meteorology and also enables this technology to also work internationally.

\subsection{Irrigation and Rainfall}

The mobile app will provide a way for irrigators to enter how much irrigation they have applied to each field, and also how much rainfall has fallen as it occurs. This information needs to be supplied by the user since most irrigation pumps are not telemeted, and rainfall from a nearby weather station is not local enough to accurately determine how much rainfall was observed on their fields. The new application user interface will provide a richer user experience, and more accurate methods of data entry over the previous SMS message approach. The application being developed will be similar in functionality to the Smartirrigation Cotton app (Vellidis et al, 2014) which has been developed for use in the United States (Fig. 6)

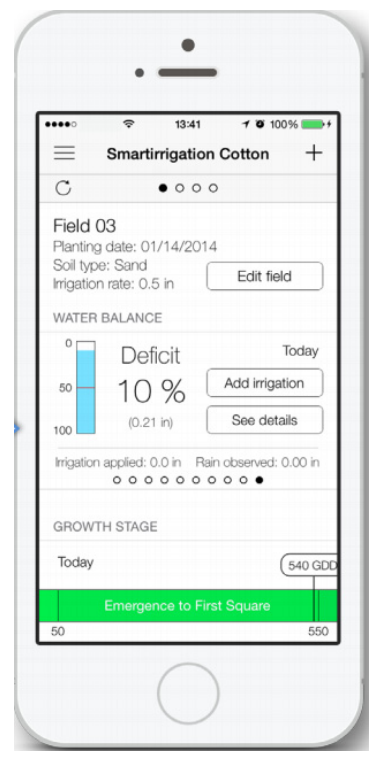

Fig. 6. Smartirrigation Cotton application developed for iOS 


\subsection{Benchmarking-Based Irrigation Decision Support}

Unlike the previous generation of IrriSAT system, this next generation will deliver advice to irrigators based initially on the water balance (rule-based) system and then, after a season of operation for baselining, advice from a benchmarking, case-based reasoning system will also be delivered.

Time-delimited periods of irrigation practice (weekly or monthly) will be treated as cases in a Case-Based Reasoning (CBR), as described by Aamodt \& Plaza (1994). The input conditions to these cases (the weather and crop data) will be used with a measure of utility of the case results (the irrigation decision(s) made within the timeframe) according to standard CBR methodology. The utility measure will be gathered from yield data at the end of the first season's operation.

From the start of the second season's operation (2015/2016) onwards, the CBR system will compare a current irrigator's situation (the 'current case' in CBR terminology) with past, similar situations (the 'case base') and provide advice in parallel with the rule-based advice. How this advice will be presented to the irrigators is future work.

\section{Conclusion}

Through the GEE case study, we have found the API to be adequate for undertaking the desired processing tasks required for determining paddock scale crop coefficients required by IrriSAT. The work from this case study is currently further being integrated into the next generation IrriSAT DSS which will be capable of: providing more regular Kc estimates than previously by using both Landsat 8 and Landsat 7 imagery; and will also provide a completely automated data processing pipeline. Adopting the GEE platform will allow us to access and run algorithms on the full Earth Engine data archive, all using Google's parallel processing platform in real time, enabling the next generation IrriSAT to easily scale across all of Australia.

Exposing the weather observations via SOS allows our applications and any other applications, to easily query and retrieve information over the web. We have currently migrated all historic weather station data into 52 north's SOS implementation. Further work involves developing automated data ingestion routines to ensure the datasets are kept up to date for near-real time accessibility.

Once the application is developed, irrigation information will be able to be collected from a limited selection of farmers throughout the first cotton growing season. This will enable us to develop the benchmarking products required to compare water usage against other cotton irrigators in nearby regions and also against previous growing seasons.

Our use of new and sophisticated datasets and web services at the national and regional (weather station) levels will enable us to avoid much of the data preparation complexity of previous generation DSS. The DSS core application will be capable of requesting information from the external data services to run a water balance calculation; whilst all of the raw data management and preparation steps generating the required products have already been undertaken by the data providers. 


\section{References}

1. Aamodt, A., Plaza, E.: Case-based reasoning: Foundational issues, methodological variations, and system approaches. AICom - Artificial Intelligence Communications 7(1), 3959 (1994)

2. Allen, R.G., Pereira, L.S., Raes, D., Smith, M.: Crop Evapotranspiration - Guidelines for Computing Crop Water Requirements. FAO Irrigation and Drainage Paper 56 (1998)

3. Car, N.J., Christen, E.W., Hornbuckle, J.W., Moore, G.A.: Using a mobile phone Short Messaging Service (SMS) for irrigation schedulingin Australia - Farmers' participation and utility evaluation. Computers and Electronics in Agriculture 84, 132-143 (2012)

4. Flood, N.: Continuity of Reflectance Data between Landsat-7 ETM+ and Landsat-8 OLI, for Both Top-of-Atmosphere and Surface Reflectance: A Study in the Australian Landscape, remote sensing (2014)

5. Gorelick, N.: Google Earth Engine,Geophysical Research Abstracts, vol. 15, EGU201311997, 2013 EGU General Assembly (2013)

6. Hornbuckle, J.W., Car, N.J., Christen, E.W., Stein, T.M., Williamson, B.: IrriSatSMS - Irrigation Water Management by Satellite and SMS - A Utilisation Framework. CRC for Irrigation Futures Technical Report No. 01/09and CSIRO Land and Water Science Report No. 04/09. CSIRO Land and Water,Griffith, NSW, Australia (2009)

7. Liaw, Y.P., Cook, D.R., Sisterson, D.L., Gao, W.: Comparison of Satellite-Derived and Observer-Based Determinations of Cloud Cover Amount at the Southern Great Plains Cloud and Radiation Testbed Site. Fifth Atmospheric Radiation Measurement San Diego, California (March 1995)

8. Vellidis, G., Liakos, V., Perry, C., Tucker, M., Collins, G., Snider, J., Andreis, J., Migliaccio, K., Fraisse, C., Morgan, K., Rowland, D., Barnes, E.: A smartphone app for scheduling irrigation on cotton. In: Boyd, S., Huffman, M., Robertson, B. (eds.) Proceedings of the 2014 Beltwide Cotton Conference, New Orleans, LA, National Cotton Council, Memphis, TN (2014) (paper 15551)

9. Zhu, Z., Woodcock, C.E.: Object-based cloud and cloud shadow detection in Landsat imagery. Remote Sensing of Environment 118, 83-94 (2012) 